\title{
JULIA SETS CONVERGING TO THE UNIT DISK
}

\author{
ROBERT L. DEVANEY AND ANTONIO GARIJO \\ (Communicated by Jane M. Hawkins)
}

\begin{abstract}
We consider the family of rational maps $F_{\lambda}(z)=z^{n}+\lambda / z^{d}$, where $n, d \geq 2$ and $\lambda$ is small. If $\lambda$ is equal to 0 , the limiting map is $F_{0}(z)=z^{n}$ and the Julia set is the unit circle. We investigate the behavior of the Julia sets of $F_{\lambda}$ when $\lambda$ tends to 0 , obtaining two very different cases depending on $n$ and $d$. The first case occurs when $n=d=2$; here the Julia sets of $F_{\lambda}$ converge as sets to the closed unit disk. In the second case, when one of $n$ or $d$ is larger than 2, there is always an annulus of some fixed size in the complement of the Julia set, no matter how small $|\lambda|$ is.
\end{abstract}

In this paper we consider the family of rational maps

$$
F_{\lambda}(z)=z^{n}+\frac{\lambda}{z^{d}}
$$

where $n, d \geq 2$ and $|\lambda|$ is small but nonzero. Our goal is to investigate the behavior of the Julia sets for this family of maps as $\lambda \rightarrow 0$.

Clearly, as $\lambda \rightarrow 0$, the maps $F_{\lambda}$ converge to $z \mapsto z^{n}$. The Julia set of this limiting map is well understood: it is the unit circle. But the situation for $F_{\lambda}$ is quite different since the degree of $F_{\lambda}$ jumps to $n+d$ as soon as $\lambda \neq 0$.

There are two very different cases to consider when $\lambda \neq 0$. The first case occurs when $n$ and $d$ are not both equal to 2 . In this case, it is known by the work of McMullen [8] that the Julia set of $F_{\lambda}$ for $\lambda$ sufficiently small is a Cantor set of simple closed curves, each of which surrounds the origin (see Figure 1). So, in particular, the Julia set is not connected when $|\lambda|$ is small. Indeed, there is a unique open disk $\mathcal{M}$ surrounding the origin in the $\lambda$-plane such that the Julia set of $F_{\lambda}$ is always a Cantor set of simple closed curves when $\lambda \in \mathcal{M}-\{0\}$ [7]. Moreover, all such maps with $\lambda \in \mathcal{M}-\{0\}$ are the same dynamically in the sense that any two such maps are conjugate on their Julia sets.

When $n=d=2$, the situation is very different. It is known [1] that there are no Julia sets of the above type in this case; in fact, all Julia sets of $F_{\lambda}$ for $|\lambda|$ small are connected sets (see Figure 2). Also, it is known that there are uncountably many dynamically different types of Julia sets in any neighborhood of the origin in parameter space. Indeed, in any neighborhood of 0 , there are infinitely many parameters for which the Julia set is a Sierpinski curve, but the dynamics of any two such maps are nonconjugate.

Received by the editors November 29, 2006.

2000 Mathematics Subject Classification. Primary 37F10, 37F40.

The second author was supported by MTM2005-02139/Consolider (including a FEDER contribution) and CIRIT 2005 SGR01028. 

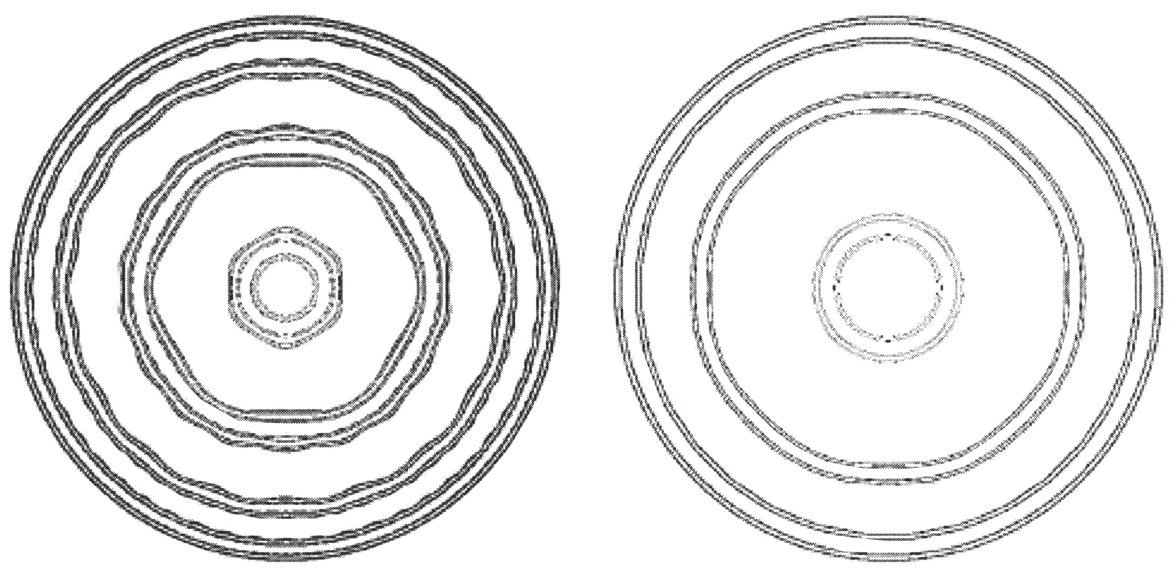

Figure 1. The Julia sets for $z^{3}-0.001 / z^{3}$ and $z^{4}-0.001 / z^{4}$ are both Cantor sets of circles.
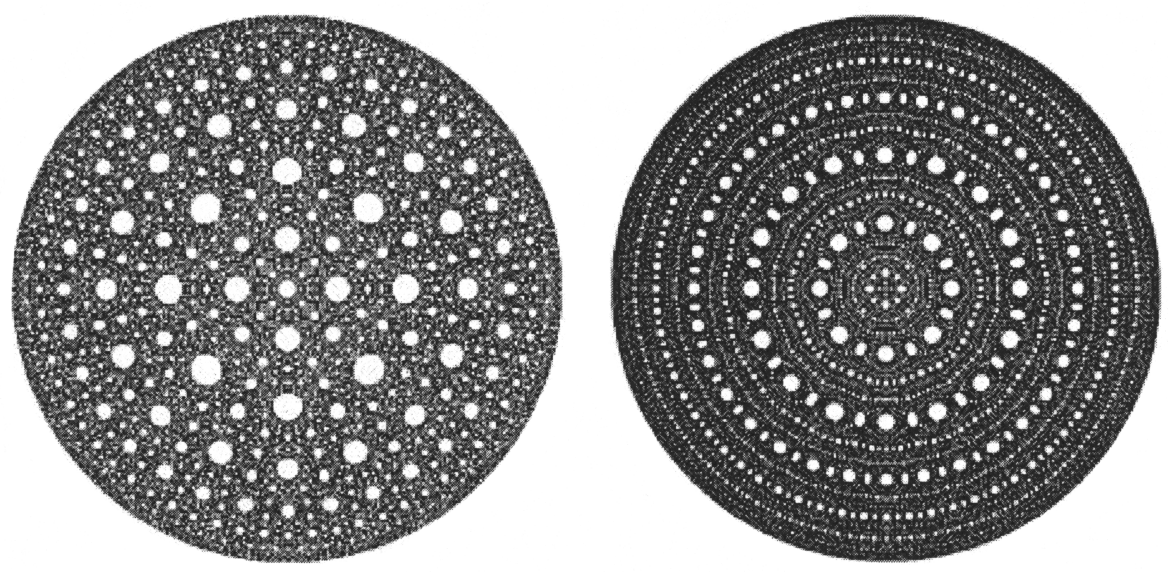

FiguRE 2. The Julia sets for $n=2$ and $\lambda=-0.001$ and $\lambda=-0.00001$.

How these two different types of Julia sets evolve as $\lambda \rightarrow 0$ is also quite different. In Figure 1, note the large white annular regions between the closed curves in the Julia sets. These annuli lie in the complement of the Julia set. As $n$ or $d$ grows, one can find even larger annuli in the complement of the Julia set, no matter how small $|\lambda|$ is. On the other hand, in Figure 2, the complementary regions in the Julia sets all seem to be disks whose radii decrease with $|\lambda|$. This is indeed the case. We shall prove that, when $n=d=2$, the Julia sets of $F_{\lambda}$ converge as sets to the closed unit disk. On the other hand, when one of $n$ or $d$ is larger than 2 , there is always an annulus of some fixed size in the complement of the Julia set, no matter how small $|\lambda|$ is. More precisely, the main result in this paper is:

Theorem 1. (1) Suppose $n=d=2$. If $\lambda_{j}$ is a sequence of parameters converging to 0 , then the Julia sets of $F_{\lambda_{j}}$ converge as sets to the closed unit disk. 
(2) If one of $n$ or $d$ is not equal to 2, this is not the case. Specifically, for a given punctured neighborhood $U$ of $0 \in \mathcal{M}$, there exists $\delta>0$ such that, for each $\lambda \in U$, there is a round annulus (i.e., bounded by circles) in the complement of the Julia set inside the unit circle whose internal and external radii differ by at least $\delta$.

\section{Julia SETS CONVERGing TO THE UNIT DISK}

In this section we restrict attention to the family of maps

$$
F_{\lambda}(z)=z^{2}+\frac{\lambda}{z^{2}}
$$

where $\lambda \neq 0$. Note that the point at $\infty$ is a superattracting fixed point. We denote the immediate basin of $\infty$ by $B_{\lambda}$. The origin is a pole of order two, so there is a neighborhood of 0 that is mapped onto a neighborhood of $\infty$ in $B_{\lambda}$. If this neighborhood is disjoint from $B_{\lambda}$, we denote the preimage of $B_{\lambda}$ containing 0 by $T_{\lambda}$. This set is known as the trap door, since any orbit that eventually enters $B_{\lambda}$ must do so by passing through the trap door.

The Julia set of $F_{\lambda}$, denoted by $J\left(F_{\lambda}\right)$, is known to be the boundary of the set of points whose orbits escape to $\infty$ (see [1]).

One computes easily that the map $F_{\lambda}$ has four critical points given by $c_{\lambda}=\lambda^{1 / 4}$. There are only two critical values, however, namely $v_{\lambda}=2 \lambda^{1 / 2}$. Furthermore, the second iterates of all of the critical points all land on the same point, namely $\omega_{\lambda}=1 / 4+4 \lambda$. Thus, there is essentially only one critical orbit for this family. Moreover, $J\left(F_{\lambda}\right), B_{\lambda}$, and $T_{\lambda}$ are all symmetric under rotation by a fourth root of unity.

Theorem 2 is proved in [1]:

Theorem 2. (1) Suppose one (and hence both) of the critical values lies in $B_{\lambda}$. Then $J\left(F_{\lambda}\right)$ is a Cantor set. Otherwise, $J\left(F_{\lambda}\right)$ is connected.

(2) Suppose that, for some $k \geq 2, F_{\lambda}^{k}\left(c_{\lambda}\right)$ lies in $B_{\lambda}$ but $F_{\lambda}^{k-1}\left(c_{\lambda}\right)$ does not lie in $B_{\lambda}$. Then the Julia set of $F_{\lambda}$ is a Sierpinski curve.

A Sierpinski curve is any planar set that is homeomorphic to the well known Sierpinski carpet fractal. Equivalently, any planar set that is compact, connected, locally connected, nowhere dense, and has the property that any pair of complementary domains are bounded by simple closed curves that are pairwise disjoint is known to be homeomorphic to the Sierpinski carpet [10]. In [5] it was shown that, in any neighborhood of the origin in the $\lambda$-plane, there are infinitely many parameters for which $J\left(F_{\lambda}\right)$ is a Sierpinski curve, but the dynamical behavior of $F_{\lambda}$ is different for each of these parameters.

Suppose now that $J\left(F_{\lambda}\right)$ is a connected set. Then it has been proved in [3] that, if $|\lambda|<1 / 16$, then the Julia set always contains an invariant Cantor necklace. A Cantor necklace is a set that is homeomorphic to the following subset of the plane: Place the Cantor middle thirds set on the $x$-axis. Then adjoin a circle of radius $1 / 3^{j}$ in place of each of the $2^{j}$ removed intervals at the $j^{\text {th }}$ level of the construction of the Cantor middle thirds set. The union of the Cantor set and the adjoined circles is the model for the Cantor necklace.

In the Julia set of $F_{\lambda}$, the invariant Cantor necklace has the following properties. The simple closed curve corresponding to largest circle in the model is the boundary of the trap door. All of the closed curves corresponding to the circles at level $j$ 
correspond to the boundaries of preimages of $\partial B_{\lambda}$ that map to this set after $j$ iterations. The Cantor set portion of the necklace is an invariant set on which $F_{\lambda}$ is hyperbolic and, in fact, conjugate to the one-sided shift map on two symbols. The two extreme points in this set correspond to the fixed point and its negative, both of which lie in $\partial B_{\lambda}$. Hence the Cantor necklace stretches completely "across" $J\left(F_{\lambda}\right)$. Moreover, it is known that the Cantor necklace is located in a particular subset of the Julia set. Specifically, let $c_{0}(\lambda)$ be the critical point of $F_{\lambda}$ that lies in the sector $0 \leq \operatorname{Arg} z<\pi / 2$ when $0 \leq \operatorname{Arg} \lambda<2 \pi$. Let $c_{j}$ be the other critical points arranged in a counterclockwise direction around the origin as $j$ increases. Let $I_{0}$ denote the sector bounded by the two straight lines connecting the origin to $\infty$ and passing through $c_{0}$ and $c_{1}$. Let $I_{1}$ be the negative of this sector. Then, as shown in [3], the Cantor set portion of the necklace is constrained to lie in this region for all $\lambda$ with $0 \leq \operatorname{Arg} \lambda<2 \pi$.

We now prove that when $n=d=2$, the Julia sets of $F_{\lambda}$ converge to the closed unit disk $\mathbb{D}$ as $\lambda \rightarrow 0$. By "converges to the unit disk" we mean the following.

Theorem 3. Let $\epsilon>0$ and denote the disk of radius $\epsilon$ centered at $z$ by $B_{\epsilon}(z)$. There exists $\mu>0$ such that, for any $\lambda$ with $0<|\lambda| \leq \mu, J\left(F_{\lambda}\right) \cap B_{\epsilon}(z) \neq \emptyset$ for all $z \in \mathbb{D}$. Also, $J\left(F_{\lambda}\right) \subset B_{1+\epsilon}(0)$.

Proof. Suppose that this is not the case. Then, given any $\epsilon>0$, we may find a sequence of parameters $\lambda_{j} \rightarrow 0$ and another sequence of points $z_{j} \in \mathbb{D}$ such that $J\left(F_{\lambda_{j}}\right) \cap B_{2 \epsilon}\left(z_{j}\right)=\emptyset$ for each $j$. Since $\mathbb{D}$ is compact, there is a subsequence of the $z_{j}$ that converges to some point $z^{*} \in \mathbb{D}$. For each parameter in the corresponding subsequence, we then have $J\left(F_{\lambda_{j}}\right) \cap B_{\epsilon}\left(z^{*}\right)=\emptyset$. Hence we may assume at the outset that we are dealing with a subsequence $\lambda_{j} \rightarrow 0$ such that $J\left(F_{\lambda_{j}}\right) \cap B_{\epsilon}\left(z^{*}\right)=\emptyset$.

Now consider the circle of radius $\left|z^{*}\right|$ centered at the origin. This circle meets $B_{\epsilon}\left(z^{*}\right)$ in an arc $\gamma$ of length $\ell$. Choose $k$ so that $2^{k} \ell \geq 2 \pi$.

Since $\lambda_{j} \rightarrow 0$, we may choose $j$ large enough so that $\left|F_{\lambda_{j}}^{i}(z)-z^{2^{i}}\right|$ is very small for $1 \leq i \leq k$, provided $z$ lies outside the circle of radius $\left|z^{*}\right| / 2$ centered at the origin. In particular, it follows that $F_{\lambda_{j}}^{k}(\gamma)$ is a curve whose argument increases by approximately $2 \pi$; i.e., the curve $F_{\lambda_{j}}^{k}(\gamma)$ wraps at least once around the origin. As a consequence, the curve $F_{\lambda_{j}}^{k}(\gamma)$ must meet the Cantor necklace in the dynamical plane. But this necklace lies in $J\left(F_{\lambda_{j}}\right)$. Hence $J\left(F_{\lambda_{j}}\right)$ must intersect this curve. Since the Julia set is backward invariant, it follows that $J\left(F_{\lambda_{j}}\right)$ must intersect $B_{\epsilon}\left(z^{*}\right)$. This then yields a contradiction, and so the result follows. The fact that $J\left(F_{\lambda}\right) \subset B_{1+\epsilon}(0)$ follows from the fact that $\partial B_{\lambda}$ tends to the unit circle as $\lambda \rightarrow 0$.

\section{The McMullen domain case}

In this section we discuss the size of the Julia sets for $F_{\lambda}(z)=z^{n}+\lambda / z^{d}$ in the case where $n, d \geq 2$ but not both $n=d=2$ and $|\lambda|$ is small. As we discussed above, in this case the Julia set is a Cantor set of simple closed curves, each of which surrounds the origin. So the Fatou set is a countable collection of annuli together with the disks $B_{\lambda}$ and $T_{\lambda}$. Following [2], we say that an annulus of the form $0<r_{1}<|z|<r_{2}$ is a round annulus. Such an annulus therefore contains a disk whose diameter is $r_{2}-r_{1}$. Our goal is to prove the following. 


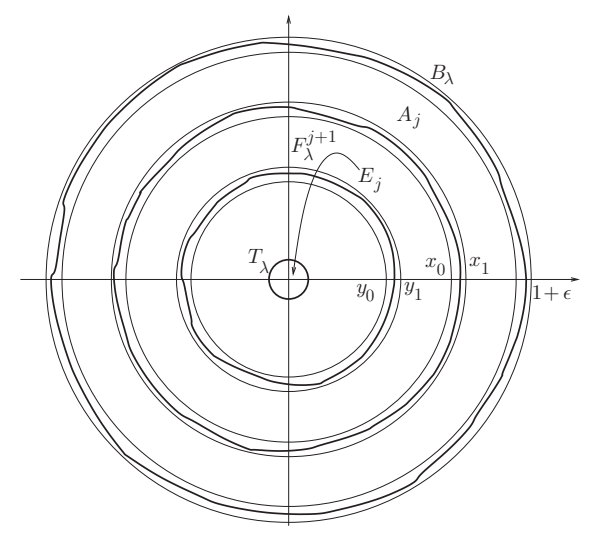

FiguRE 3. A sketch of the relevant objects in the McMullen domain case.

Theorem 4. Given $n$ and $d$, there exists $\mu, \delta>0$ such that, for all $\lambda$ with $|\lambda| \leq \mu$, one of the complementary annuli in the Fatou set of $F_{\lambda}$ contains a round annulus whose inner and outer radii differ by at least $\delta$.

As a consequence of this result, we have a very different situation when $\lambda \rightarrow 0$ in this case: the Julia sets no longer converge to the unit disk as in the case $n=d=2$.

For clarity, we shall restrict ourselves at first to the case where $n=3$; we discuss the rather straightforward modifications necessary to handle the more general case at the end of this section. So, for now, let $F_{\lambda}(z)=z^{3}+\lambda / z^{3}$, where $\lambda$ belongs to the McMullen domain.

Let $\mathcal{A}$ denote the open annulus separating $\overline{T_{\lambda}}$ from $\overline{B_{\lambda}}$. There are three disjoint open annuli lying in $\mathcal{A}$. The first is the preimage of $T_{\lambda}$ under $F_{\lambda}$ which we denote by $E_{0}$. This is the escape annulus. The region lying between $\overline{B_{\lambda}}-$ and $\overline{E_{0}}$ is an open annulus that we denote by $A_{0}$, and the region lying between $\overline{T_{\lambda}}$ and $\overline{E_{0}}$ is also an open annulus that we denote by $A_{0}^{\prime}$. Note that each of these three annuli is bounded by a simple closed curve that is mapped to either $\partial B_{\lambda}$ or $\partial T_{\lambda}$. So these annuli are disjoint but their closures fill the region between $B_{\lambda}$ and $T_{\lambda}$.

Let $m=m_{\lambda}$ denote the modulus of $\mathcal{A}$. Since the trap door shrinks to the origin as $\lambda \rightarrow 0$, it follows that $m_{\lambda} \rightarrow \infty$ as $\lambda \rightarrow 0$. Now $F_{\lambda}$ maps $E_{0}$ onto $T_{\lambda}$ whereas $F_{\lambda}$ takes both $A_{0}$ and $A_{0}^{\prime}$ onto $\mathcal{A}$ as a three-to-one covering map. Hence the modulus of both $A_{0}$ and $A_{0}^{\prime}$ is $m / 3$. The complementary annulus, namely $E_{0}$, thus also has modulus $m / 3$.

Since $F_{\lambda}$ takes $A_{0}$ onto $\mathcal{A}$ as a three-to-one covering, there are preimages of $A_{0}$, $E_{0}$, and $A_{0}^{\prime}$ in $A_{0}$. Denote these preimages by $A_{1}, E_{1}$, and $A_{1}^{\prime}$, respectively. Then the modulus of each of these annuli is $m / 3^{2}$. Now we continue inductively. Since $F_{\lambda}$ maps $A_{j}$ as a three-to-one covering over $A_{j} \cup E_{j} \cup A_{j}^{\prime}$, there are three disjoint annuli in $A_{j}$ called $A_{j+1}, E_{j+1}$, and $A_{j+1}^{\prime}$, respectively; these annuli are mapped to their predecessors. Hence the modulus of each of these annuli is $m / 3^{j+2}$. We have that $F_{\lambda}^{j+1}$ maps $E_{j}$ onto $T_{\lambda}$, so each $E_{j}$ lies in the Fatou set (see Figure 3 ). We shall show that, for each sufficiently small $\lambda$, there is an $E_{j}$ that contains a round annulus which contains disks whose diameter is uniformly bounded away from 0 . 
We now complete the proof of Theorem 4 in the case $n=3$. Since $m=m_{\lambda} \rightarrow \infty$ as $\lambda \rightarrow 0$, we may find a $j \geq 0$ that depends on $\lambda$ such that

$$
1<\bmod A_{j}=\bmod E_{j} \leq 3 .
$$

Let $\alpha=\alpha(\lambda)$ denote the modulus of $A_{j}$ and $E_{j}$. So $1<\alpha(\lambda) \leq 3$ for all sufficiently small $\lambda$. We emphasize that $j$ increases as $\lambda \rightarrow 0$, but there always exist $A_{j}$ and $E_{j}$ with moduli between 1 and 3 .

Given any $\epsilon$ with $0<\epsilon<1$, let $x_{0}$ satisfy

$$
\frac{1}{2 \pi} \log \left(\frac{1-\epsilon}{x_{0}}\right)=\alpha
$$

so that $x_{0}=(1-\epsilon) \exp (-2 \pi \alpha)$. Similarly, let $x_{1}$ satisfy

$$
\frac{1}{2 \pi} \log \left(\frac{1+\epsilon}{x_{1}}\right)=\alpha
$$

so that $x_{1}=(1+\epsilon) \exp (-2 \pi \alpha)$. Also let $y_{0}$ and $y_{1}$ satisfy

$$
\frac{1}{2 \pi} \log \left(\frac{1-\epsilon}{y_{0}}\right)=2 \alpha
$$

and

$$
\frac{1}{2 \pi} \log \left(\frac{1+\epsilon}{y_{1}}\right)=2 \alpha,
$$

respectively, so that $y_{0}=(1-\epsilon) \exp (-4 \pi \alpha)$ and $y_{1}=(1+\epsilon) \exp (-4 \pi \alpha)$. We assume here that $\epsilon$ is small enough so that

$$
\exp (-4 \pi \alpha)(1+\epsilon)<\exp (-2 \pi \alpha)(1-\epsilon)<\exp (-2 \pi \alpha)(1+\epsilon)<1-\epsilon .
$$

Therefore, we have

$$
y_{0}<y_{1}<x_{0}<x_{1}<1-\epsilon
$$

for sufficiently small $\lambda$ (see Figure 3 for a sketch of the construction above). Observe that, since $1<\alpha \leq 3$, we have that each of the $x_{i}$ and $y_{i}$ lie in a compact interval in the open interval $(0,1)$ and, in particular, these points are strictly bounded away from 0 .

Since $\partial B_{\lambda}$ tends to the unit circle as $\lambda \rightarrow 0$, we may choose $\mu>0$ so that, if $|\lambda| \leq \mu$, then we have that $\partial B_{\lambda}$ lies in the round annulus given by $1-\epsilon<|z|<1+\epsilon$.

Now consider the position of $A_{j}$. Since $\bmod A_{j}=\alpha$ and the outer boundary of $A_{j}$ is $\partial B_{\lambda}$ (and so lies outside the circle $|z|=1-\epsilon$ ), we have that the inner boundary of $A_{j}$ cannot lie strictly inside the circle $|z|=x_{0}$. Indeed, if that were the case, we would have

$$
\bmod A_{j}>\frac{1}{2 \pi} \log \left(\frac{1-\epsilon}{x_{0}}\right)=\alpha,
$$

which is a contradiction. On the other hand, the inner boundary of $A_{j}$ cannot lie strictly outside the circle $|z|=x_{1}$ for otherwise we would have

$$
\bmod A_{j}<\frac{1}{2 \pi} \log \left(\frac{1+\epsilon}{x_{1}}\right)=\alpha,
$$

again a contradiction. 
Now let $X_{j}$ be the open annulus $A_{j} \cup E_{j}$ together with the simple closed curve separating them. Note that $\bmod X_{j}=2 \alpha$. The inner boundary of $X_{j}$ cannot lie strictly inside the circle $|z|=y_{0}$ for we would then have

$$
\bmod \left(X_{j}\right)>\frac{1}{2 \pi} \log \left(\frac{1-\epsilon}{y_{0}}\right)=2 \alpha=\bmod X_{j} .
$$

Now consider the annulus $E_{j}$. By the above, the inner boundary of $E_{j}$ cannot lie strictly inside the circle $|z|=y_{0}$. Also, by the above, the outer boundary of $E_{j}$ cannot lie strictly outside the circle $|z|=x_{1}$ since the inner boundary of $A_{j}$ has this property. By [2], $E_{j}$ must contain a round annulus of modulus at least $\alpha-1 / 2>1 / 2$. Therefore, this round annulus must be contained in the annulus given by $y_{0}<|z|<x_{1}$. Hence the difference between the inner and outer radii of this round annulus is bounded away from 0 . This provides the $\delta$ in Theorem 4 .

Finally, we complete the proof in the McMullen domain case for all values $n, d \geq 2$ but not both $n=d=2$ and $|\lambda|$ small. In this general case all the dynamical objects are defined in the same way as in Theorem 4 , but now the moduli of the rings depend on $n$ and $d$.

As before, let $m=m_{\lambda}$ be the modulus of $\mathcal{A}$. Now $F_{\lambda}$ maps $A_{0}$ onto $\mathcal{A}$ as a $n$-to- 1 covering map and maps $A_{0}^{\prime}$ onto $\mathcal{A}$ as a $d$-to- 1 covering map. Hence the moduli of $A_{0}$ and $A_{0}^{\prime}$ are $m / n$ and $m / d$, respectively. The complementary annulus, denoted by $E_{0}$, has modulus $m(1-1 / n-1 / d)$, since

$$
\bmod (\mathcal{A})=\bmod \left(A_{0}^{\prime}\right)+\bmod \left(E_{0}\right)+\bmod \left(A_{0}\right) .
$$

Inductively, we denote by $A_{j+1}, E_{j+1}$ and $A_{j+1}^{\prime}$ the preimage of $A_{j}, E_{j}$ and $A_{j}^{\prime}$ in $A_{j}$. Since $F_{\lambda}$ maps $A_{j}$ as an $n$-to- 1 covering map over $A_{j} \cup E_{j} \cup A_{j}^{\prime}$, we have that the moduli of $A_{j+1}, E_{j+1}$ and $A_{j+1}^{\prime}$ are $\bmod \left(A_{j}\right) / n, \bmod \left(E_{j}\right) / n$ and $\bmod \left(A_{j}^{\prime}\right) / n$, respectively. Taking this into account, we obtain that

$$
\begin{aligned}
\bmod \left(A_{j+1}\right) & =\frac{\bmod \left(A_{0}\right)}{n^{j+1}}=\frac{m}{n^{j+2}}, \\
\bmod \left(E_{j+1}\right) & =\frac{\bmod \left(E_{0}\right)}{n^{j+1}}=\frac{m}{n^{j+2}}\left(1-\frac{1}{n}-\frac{1}{d}\right), \\
\bmod \left(A_{j+1}^{\prime}\right) & =\frac{\bmod \left(A_{0}^{\prime}\right)}{n^{j+1}}=\frac{m}{d n^{j+1}},
\end{aligned}
$$

for each $j \geq 0$. As before, $m=m_{\lambda} \rightarrow \infty$ when $\lambda$ tends to 0 , so we can choose $j$ such that

$$
1<\bmod \left(E_{j}\right) \leq n\left(1-\frac{1}{n}-\frac{1}{d}\right)
$$

Note that, for this value of $j$, we have that $1<\bmod \left(A_{j}\right) \leq n$, since $0<\left(1-\frac{1}{n}-\frac{1}{d}\right)<$ 1 for $n, d \geq 2$ but not both $n=d=2$.

We denote by $\alpha=\alpha(\lambda)$ and $\beta=\beta(\lambda)$ the modulus of $A_{j}$ and $E_{j}$, respectively. Given any $\epsilon$ with $0<\epsilon<1$, we define the following four auxiliary values: $x_{0}=$ $(1-\epsilon) \exp (-2 \pi \alpha), x_{1}=(1+\epsilon) \exp (-2 \pi \alpha), y_{0}=(1-\epsilon) \exp (-2 \pi(\alpha+\beta))$, and $x_{0}=(1+\epsilon) \exp (-2 \pi(\alpha+\beta))$. Using the same argument as in Theorem $4, E_{j}$ contains a round annulus of modulus at least $\beta-1 / 2>1 / 2$. Therefore, this round annulus must be contained in the round annulus given by $y_{0}<|z|<x_{1}$. 


\section{ACKNOWLEDGMENTS}

The second author would like to thank the Department of Mathematics at Boston University for their hospitality while this work was in progress.

\section{REFERENCES}

[1] Blanchard, P., Devaney, R. L., Look, D. M., Seal, P., and Shapiro, Y. Sierpinski Curve Julia Sets and Singular Perturbations of Complex Polynomials. To appear in Ergodic Theory and Dynamical Systems. MR2158396 (2006d:37087)

[2] Ble, G., Douady, A., and Henriksen, C. Round Annuli. Contemporary Mathematics. 355 (2004), 71-76. MR2145056 (2006b:31004)

[3] Devaney, R. L. Cantor Necklaces and Structurally Unstable Sierpinski Curve Julia Sets for Rational Maps. To appear in Qual. Theory Dynamical Systems. MR2275444

[4] Devaney, R. L. Structure of the McMullen Domain in the Parameter Space of Rational Maps. Fundamenta Mathematicae. 185 (2005), 267-285. MR2161407 (2006c:37046)

[5] Devaney, R. L. and Look, D. M. A Criterion for Sierpinski Curve Julia Sets. To appear in Topology Proceedings. MR2280665

[6] Devaney, R. L., Look, D. M., and Uminsky, D. The Escape Trichotomy for Singularly Perturbed Rational Maps. Indiana U. Math. J. 54 (2005), 1621-1634. MR2189680 (2006i:37105)

[7] Devaney, R. L. and Marotta, S. The McMullen Domain: Rings Around the Boundary. To appear in Trans. Amer. Math. Soc.

[8] McMullen, C. Automorphisms of Rational Maps. Holomorphic Functions and Moduli. Vol. 1. Math. Sci. Res. Inst. Publ. 10. Springer, New York, 1988. MR955807 (89m:58187)

[9] Milnor, J. Dynamics in One Complex Variable. Vieweg, 1999. MR1721240 (2002i:37057)

[10] Whyburn, G. T. Topological Characterization of the Sierpinski Curve. Fund. Math. 45 (1958), 320-324. MR0099638 (20:6077)

Department of Mathematics, Boston University, 111 Cummington Street, Boston, MassachusetTs 02215

E-mail address: bob@bu.edu

Dep. D'Eng. Informàtica i Matemàtiques, Universitat Rovira i Virgili, Av. Països Catalans, 26, 43007 Tarragona, Spain 